\section{OPEN ACCESS}

Edited by:

Gary Iwamoto,

University of Illinois at

Urbana-Champaign, USA

Reviewed by:

Hoda Yeganehjoo,

University of Texas Southwestern

Medical Center, USA

Lona F. Sandon,

University of Texas Southwestern

Medical Center, USA

*Correspondence:

Marciane Magnani

magnani2@gmail.com.br

Specialty section

This article was submitted to

Exercise Physiology,

a section of the journal

Frontiers in Physiology

Received: 10 October 2016 Accepted: 15 March 2017

Published: 31 March 2017

Citation:

Barbosa CVdS, Silva AS, de

Oliveira CVC, Massa NML, de

Sousa YRF, da Costa WKA, Silva AC

Delatorre $P$, Carvalho R, Braga VdA

and Magnani M (2017) Effects of

Sesame (Sesamum indicum L.) Supplementation on Creatine Kinase,

Lactate Dehydrogenase, Oxidative

Stress Markers, and Aerobic Capacity in Semi-Professional Soccer Players.

Front. Physiol. 8:196.

doi: 10.3389/fphys.2017.00196

\title{
Effects of Sesame (Sesamum indicum L.) Supplementation on Creatine Kinase, Lactate Dehydrogenase, Oxidative Stress Markers, and Aerobic Capacity in Semi-Professional Soccer Players
}

Carlos V. da Silva Barbosa ${ }^{1}$, Alexandre S. Silva ${ }^{1}$, Caio V. C. de Oliveira ${ }^{1}$, Nayara M. L. Massa ${ }^{1}$, Yasmim R. F. de Sousa ${ }^{2}$, Whyara K. A. da Costa ${ }^{2}$, Ayice C. Silva ${ }^{3}$, Plínio Delatorre ${ }^{4}$, Rhayane Carvalho ${ }^{2}$, Valdir de Andrade Braga ${ }^{5}$ and Marciane Magnani ${ }^{2 *}$

${ }^{1}$ Laboratory for Physical Training Studies Applied to Performance and Health, Federal University of Paraíba, João Pessoa, Brazil, ${ }^{2}$ Laboratory of Biochemistry of Foods, Department of Food Engineering, Federal University of Paraíba, João Pessoa, Brazil, ${ }^{3}$ Embrapa Cotton, Campina Grande, Brazil, ${ }^{4}$ Department of Molecular Biology, Federal University of Paraíba, João Pessoa, Brazil, ${ }^{5}$ Biotechnology Center, Federal University of Paraiba, Joao Pessoa, Brazil

Nutritional intervention with antioxidants rich foods has been considered a strategy to minimize the effects of overtraining in athletes. This experimental, randomized, and placebo-controlled study evaluated the effects of consumption of sesame (Sesamum indicum L.) on muscle damage markers, oxidative stress, systemic inflammation, and aerobic performance in male semi-professional soccer players. Twenty athletes were randomly assigned to groups that received $40 \mathrm{~g}$ (two tablespoons) per day of sesame or a placebo during 28 days of regular training (exposed to routine training that includes loads of heavy training in the final half of the season). Before and after intervention, creatine kinase $(C K)$, lactate dehydrogenase $(\mathrm{LDH})$, malondialdehyde (MDA), superoxide dismutase (SOD), C-reactive protein (hs-CRP), and aerobic capacity were evaluated. Before intervention, a physiologic imbalance was noted in both groups related to CK and LDH levels. Sesame intake caused a reduction of CK (19\%, $p<0.05)$, LDH (37\%, $p<0.05), \operatorname{MDA}(55 \%, p<0.05)$ and hs-CRP $(53 \%, p<0.05)$ and increased SOD $(14 \%$, $p<0.05)$, vitamin A (25\%, $p<0.05)$, and vitamin $E(65 \%, p<0.05)$ in the experimental group. These phenomena were accompanied by increased aerobic capacity (17\%, $p<0.05)$. The placebo group showed an increase in CK $(5 \%, p<0.05)$ and no significant change in LDH, SOD or vitamin A. MDA levels decreased $(21 \%, p<0.05)$ and vitamin $E$ increased $(14 \%, p<0.05)$ in the placebo group, but to a much lesser extent than in the experimental group. These results show that sesame consumption may reduce muscle damage and oxidative stress while improving the aerobic capacity in soccer players.

Keywords: athletes, oxidative stress, muscle damage, inflammation, functional food 


\section{INTRODUCTION}

To achieve maximum performance, athletes often face highintensity, and/or high-volume physical training loads. Though this workout regime leads to high performance, it can also expose athletes to a non-functional risk of overreaching or overtraining. In such situations, an athlete's body cannot assimilate the training load and will show no improvement in performance or a sharp decline associated with neuroimmune endocrine disorders that affect athletes' health (Smith, 2004). In fact, it is estimated that even in young athletes, at least $20-30 \%$ have already experienced non-functional overreaching or signs of overtraining (Winsley and Matos, 2011).

Physical training naturally promotes damage of muscle tissues, which is accompanied by local inflammation. In the recovery period, muscle tissue repair and improvement of trained physical abilities occurs through the well-known phenomenon of general adaptation syndrome, which comprises non-specific systemic reactions of the body to continuous exposure to stress (Goldstein and Kopin, 2007; Aptekmann and Cesar, 2010; Winsley and Matos, 2011). However, if training loads are of high intensity and/or volume or the recovery period is inadequate, there may be an evolution from local muscle inflammation to systemic inflammation and oxidative stress, which evolves into neuroendocrine and behavioral disorders (Miranda-Vilela et al., 2009). Moreover, previous studies have shown minimization of muscle fatigue, systemic inflammation (Miranda-Vilela et al., 2009), and oxidative stress (Toscano et al., 2015) in athletes who received nutritional intervention with orange juice, pequifruit pulp oil, or grape juice, foods known for their antioxidant properties.

Sesame cultivar (Sesamum indicum L.) has high nutritional value due the significant amounts of dietary fiber, protein, natural antioxidants, unsaturated fatty acids, vitamins, and mineral constituents present in its composition (Anilakumar et al., 2010). The lipid fraction of sesame seeds shows good oxidative stability, which is attributed to the essential fatty acids, such as oleic, linoleic, and arachidonic present in its composition, as well as to antioxidant compounds (e.g., sesamin, sesamolin, and sesamol) that may act in synergism (Namiki, 2007; Kanu et al., 2010).

Previous study have demonstrated the antioxidant effects of sesame in hypertension model (Kamal-Eldin et al., 2011). Furthermore, the anti-inflammatory and antioxidant effects of sesame the anti-inflammatory and antioxidant effects of sesame via decreases in lipid peroxidation, C-reactive protein (hs-CRP), and interleukin-6 (IL-6) have also been shown (Haghighian et al., 2015). To date, whether soccer players would benefit from the antioxidant and anti-inflammatory effects of sesame has not been investigated. The aim of this study was to test the effect of a supplementation protocol with white sesame seeds (S. indicum L. cultivar BRS silk) on muscle damage markers, systemic inflammation, oxidative stress and aerobic capacity in soccer players exposed to routine training that includes loads of heavy training in the final half of the season.

\section{METHODS}

\section{Subjects}

A group of 20 male semi-professional soccer players were randomly divided into an experimental $(n=10$, age range $16-18$ years) and a placebo group ( $n=10$, average range $16-18$ years). The training routine consisted of weekly workouts of 6 days for $3 \mathrm{~h}$ per day. The exclusion criteria were articular problems, regular consumption of sesame, regular consumption of alcohol, smoking, lactose intolerance, use of medications for chronic diseases, and intake of minerals, vitamins, anabolic steroids, or similar compounds. The study was approved by the Committee on Ethical Research Involving Humans Beings of the Federal University of Paraíba (João Pessoa, Brazil) under the Protocol Number 04308612.2.0000.5183. All athletes had the permission of their legal guardian, who signed the informed consent as required by Resolution 196/96 of the Brazilian National Health Council (CNS).

\section{Experimental Design}

This was an experimental, randomized, and placebo-controlled study. After 10 months of training and games and 35 days before the last competition of the season, the athletes were submitted to the supplementation protocol with a sesame paste or placebo immediately before and after each training session for 28 days. Before and $48 \mathrm{~h}$ after the 28-day protocol, they performed tests for assessing aerobic capacity, and blood samples were collected to analyze the creatine kinase (CK), lactate dehydrogenase (LDH), high-sensitivity C-reactive protein (hsCRP), malondialdehyde (MDA), superoxide dismutase (SOD), and vitamins $\mathrm{A}$ and $\mathrm{E}$.

\section{Training History}

In the 10 months prior to the start of the study, athletes participated in regular soccer training consisting of a brief preseason aimed at the development of overall strength and aerobic endurance, followed by a regular season, during which the training focused on technical and tactical skills, strength, and speed. At the beginning of the study, athletes were preparing for a competition to be held 35 days after the beginning of the study. They had no official competitions during this period, only weekly friendly matches. During the supplementation period, all athletes maintained the regular season training schedule, which focused on the development of tactics, explosive strength and speed. The training consisted of one or two daily sessions. The athletes did not exercise in the $48 \mathrm{~h}$ preceding each blood collection and aerobic test to eliminate the acute effects of exercise.

\section{Preparation and Administration of Sesame Paste and Placebo}

Seeds of the white sesame cultivar BRS silk (EMBRAPA-Brazil) were roasted at $105^{\circ} \mathrm{C}$ for $2 \mathrm{~min}$ and then crushed in a highspeed blender $\left(\right.$ Oster ${ }^{\circledR}$ ) for $10 \mathrm{~min}$. To facilitate consumption, $40 \mathrm{~g}$ of crushed seeds was mixed into a paste with $17 \mathrm{~g}$ of honey (Apis mellifera). The placebo sesame paste had similar physical characteristics as the sesame paste and had equal caloric content. It was made using $17 \mathrm{~g}$ of honey, maltodextrin, cow milk, and 
artificial caramel food coloring (Otkcer ${ }^{\circledR}$, São Paulo, Brazil). The placebo and sesame pastes were placed in identical aluminum packages so that neither would be suspected of being the placebo. No differences between the texture and color of the placebo and sesame paste were detected in preliminary sensory studies (triangular test) performed with 60 students and professors at the Health Center of the Federal University of Paraíba (data not shown). The physicochemical composition according to the AOAC (AOAC, 2012) of the sesame paste was moisture 9.96/100 $\mathrm{g}$, soluble fibers $19.35 / 100 \mathrm{~g}$, insoluble fibers $1.88 / 100 \mathrm{~g}$, ash $3.35 / 100 \mathrm{~g}$; fat $51.58 / 100 \mathrm{~g}$; and protein $20.88 / 100 \mathrm{~g}$.

The athletes consumed $40 \mathrm{~g}$ (equivalent to two tablespoons) of sesame paste; this dose was based on a previous report on the benefits of sesame (Alipoor et al., 2012; Suwimol et al., 2012). The daily dose of sesame or placebo was consumed in two parts, one part before, and one part after one of the daily training sessions, except on game days, when the entire dose was administered just before game time. The researchers followed the training and managed the supplementation every day. Over the intervention period, the athletes were advised to maintain their dietary habits. A 24-h recall food survey was conducted weekly by trained interviewer (three times: 2 weekdays and 1 weekend day) to obtain information regarding food intake (Toscano et al., 2015). The average dietary intake was used to calculate the intake of nutrients using Avanutri Revolution software, version 4.0 (Avanutri, Rio de Janeiro, Brazil). Functional tests were performed to determine the running speed at the lactate threshold of $3.5 \mathrm{mM}(\mathrm{V} 3.5 \mathrm{mM})$ and the peak velocity and peak oxygen consumption (peak VO2) following the Bruce protocol (Bruce et al., 1973) $48 \mathrm{~h}$ after the final training.

\section{Biochemical Tests}

After $12 \mathrm{~h}$ of fasting and before any exercise was performed that day, a blood sample $(10 \mathrm{~mL})$ was collected from the antecubital vein and centrifuged at 3,000 rpm for $15 \mathrm{~min}$. The serum was collected and stored at $-80^{\circ} \mathrm{C}$ for analysis of serum creatine kinase (CK), lactate dehydrogenase (LDH), malondialdehyde (MDA), and high-sensitivity C-reactive protein (hs-CRP). The CK and LDH enzymes were measured in the serum using the Labtest kit (Minas Gerais, Brazil) according to the manufacturer's instructions. The reading was performed in a Labmax Plenno at a wavelength of $340 \mathrm{~nm}$. The SOD activity was determined by a RANSOD kit (Randox, USA) with readings of the red formazan performed with superoxide radical in a spectrophotometer at $505 \mathrm{~nm}$ following the manufacturer's instructions. MDA in the serum was determined using trichloroacetic acid, acid hydrolysis, and formation of a MDA-thiobarbituric acid complex. The MDA-(TBA)2 adduct was separated from other interfering compounds by C-18 reverse-phase HPLC techniques, with visible detection at $532 \mathrm{~nm}$ (Templar et al., 1999). The serum levels of vitamins A and $\mathrm{E}$ were measured using high-performance liquid chromatography (Dionex Ultimate 3000; Thermo Scientific, Mass., USA) at 325 $\mathrm{nm}$ for the quantification of vitamin A (retinol) and $295 \mathrm{~nm}$ for the quantification of vitamin E ( $\alpha$-tocopherol). The hs-CRP was assayed by immune turbidimetry using an automatic biochemical analyzer, Cobas Mira Plus (Roche), and the PCR Reagent Plus Ultra Turbiquest at $540 \mathrm{~nm}$.

\section{Statistical Analysis}

The data are expressed as the mean and standard deviation of the mean. The independent $T$-test was performed to compare the initial values of the experimental and control groups. Twoway ANOVA (treatment by time) was performed to compare the differences between the treatments, considering $p \leq 0.05$. All of the procedures were performed using GraphPad Prism 3.4 for Windows (GraphPad Software, San Diego, CA, USA).

\section{RESULTS}

Athletes in the experimental and placebo groups presented no differences regarding the assessed anthropometric variables (Table 1). The CK, LDH, MDA, and hs-CRP values obtained before the experimental intervention suggested physiological imbalances in the athletes included in the study, but no differences between groups were observed for any of these variables. SOD values were significantly lower in the experimental group than in the control group (Table 1).

The weekly $24-\mathrm{h}$ recalls showed that the two groups were similar with respect to nutritional intake (Table 2), and no differences were observed in the consumption of calories or of compounds recognized as antioxidants, such as vitamin $\mathrm{C}$ ( $\pm 77 \mathrm{mg} /$ day $)$, vitamin $\mathrm{E}( \pm 11 \mathrm{mg} /$ day $)$, or vitamin $\mathrm{A}$ ( $\pm 790 \mathrm{mg} /$ day). However, both groups demonstrated an initial deficiency in intake of vitamin A and vitamin E based on the recommendations of the DRI (Institute of Medicine, 2004).

There were no reports of adverse symptoms such as bloating, cramping, nausea, vomiting, or diarrhea during the intervention,

TABLE 1 | Baseline characteristics of the groups before the intervention period.

\begin{tabular}{|c|c|c|c|}
\hline $\begin{array}{l}\text { Anthropometric } \\
\text { variables }\end{array}$ & $\begin{array}{l}\text { Experimental group } \\
\qquad(n=10)\end{array}$ & $\begin{array}{l}\text { Placebo group } \\
\qquad(n=10)\end{array}$ & $P$-value* \\
\hline Weight (kg) & $70.08 \pm 4.52$ & $68.02 \pm 5.73$ & 0.393 \\
\hline Height (cm) & $179.60 \pm 7.47$ & $174.10 \pm 6.04$ & 0.103 \\
\hline Body mass index $\left(\mathrm{kg} / \mathrm{m}^{2}\right)$ & $21.77 \pm 1.51$ & $22.45 \pm 1.46$ & 0.335 \\
\hline Body fat (\%) & $12.45 \pm 2.14$ & $13.76 \pm 1.98$ & 0.189 \\
\hline \multicolumn{4}{|l|}{ FUNCTIONAL TESTS } \\
\hline Peak $\mathrm{VO}_{2}\left(\mathrm{ml} \cdot \mathrm{kg}^{-1} \cdot \mathrm{min}^{-1}\right)$ & $52.47 \pm 4.10$ & $52.61 \pm 4.71$ & 0.557 \\
\hline Peak velocity $\left(\mathrm{km} \cdot \mathrm{h}^{-1}\right)$ & $16.00 \pm 1.33$ & $16.05 \pm 0.71$ & 0.589 \\
\hline $\mathrm{V}_{3.5 \mathrm{mM}}\left(\mathrm{km} \cdot \mathrm{h}^{-1}\right)$ & $12.02 \pm 2.01$ & $12.04 \pm 2.33$ & 0.716 \\
\hline \multicolumn{4}{|l|}{ BIOCHEMICAL MARKERS } \\
\hline CK (U/L) & $568.20 \pm 32.18$ & $515.90 \pm 43.340$ & 0.024 \\
\hline LDH (U/L) & $537.40 \pm 193.10$ & $516.10 \pm 231.40$ & 0.850 \\
\hline MDA ( $\mu \mathrm{mol} / \mathrm{L})$ & $1.80 \pm 1.00$ & $1.90 \pm 0.500$ & 0.811 \\
\hline SOD (U/ml) & $58 \pm 1.50$ & $60 \pm 2.00$ & 0.018 \\
\hline hs-CRP (mg/dL) & $0.366 \pm 0.14$ & $0.331 \pm 0.18$ & 0.683 \\
\hline Vitamin A ( $\mu \mathrm{g} / \mathrm{dL})$ & $36.3 \pm 3.1$ & $37.2 \pm 2.2$ & 0.703 \\
\hline Vitamin E ( $\mu \mathrm{g} / \mathrm{dL})$ & $9.8 \pm 1.8$ & $10.5 \pm 1.1$ & 0.596 \\
\hline
\end{tabular}


and no changes occurred in body mass index and body fat of the athletes, as assessed by skinfold measures.

The intake of sesame paste caused a significant decrease in serum CK levels of $\sim 19 \%$ in the experimental group relative to the initial values. At the end of the assessed period, serum CK in the experimental group was $5 \%$ lower than in the placebo group $(P<0.0001$; Figure 1A). CK levels were higher in the

TABLE 2 | Food intake of the athletes involved in the study.

\begin{tabular}{lccc}
\hline Variables & $\begin{array}{c}\text { Experimental group } \\
(\boldsymbol{n}=\mathbf{1 0})\end{array}$ & $\begin{array}{c}\text { Placebo group } \\
(\boldsymbol{n}=\mathbf{1 0})\end{array}$ & $\boldsymbol{P}$-value \\
\hline Total calories (Kcal) & $2470 \pm 810.4$ & $2993 \pm 703.7$ & 0.075 \\
Carbohydrates (g/kg/day) & $5.13 \pm 1.6$ & $6.53 \pm 2.18$ & 0.060 \\
Carbohydrates (\%) & $58.44 \pm 4.13$ & $57.71 \pm 4.36$ & 0.643 \\
Protein (g/kg/day) & $1.39 \pm 0.43$ & $1.59 \pm 0.39$ & 0.198 \\
Protein (\%) & $14.92 \pm 2.69$ & $14.78 \pm 3.03$ & 0.895 \\
Lipids (g/kg/day) & $1.06 \pm 0.42$ & $1.35 \pm 0.36$ & 0.057 \\
Lipids (\%) & $26.64 \pm 3.54$ & $27.61 \pm 3.68$ & 0.471 \\
Vitamin A (RE) & $* 789.8 \pm 148.0$ & $* 698.4 \pm 421.2$ & 0.438 \\
Vitamin C (mg) & $677.5 \pm 650.3$ & $486 \pm 415.3$ & 0.434 \\
Vitamin E (mg) & ${ }^{*} 9.73 \pm 5.15$ & ${ }^{*} 10.95 \pm 6.3$ & 0.568
\end{tabular}

Recommended daily dietary nutrient antioxidants by DRI's (2002/2005): Vitamin A:

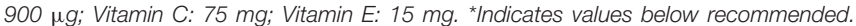
Dados are described as average \pm standard deviation of the average. placebo group at the end of the experiment $(515.2 \pm 15.81$ $\mathrm{U} / \mathrm{L})$ than at the beginning $(584.3 \pm 10.48 \mathrm{U} / \mathrm{L})$. Athletes who consumed the sesame paste showed a significant decrease in $\mathrm{LDH}$ enzyme at the end of the intervention period with $P=$ 0.0184 (37\% lower than baseline; Figure 1B). The placebo group showed no change in this variable, but the final values for the experimental group were only descriptively lower than the values found in the placebo group. Both groups showed a significant reduction in hs-CRP between the beginning and end of the study (experimental group $P=0.0006$; placebo group $P=0.0020$ ), with no differences between the groups at the end of the study (Figure 1C). MDA levels in the experimental group decreased significantly compared to baseline ( $P=0.0144$; Figure 2A). The placebo group also showed a significant reduction of MDA $(\mathrm{P}=0.0091)$, but the reduction observed in the experimental group was greater ( $\sim 55$ vs. $21 \%)$, and supplemented athletes completed the study with values significantly lower than in the placebo group $(0.7 \pm 0.07 \mu \mathrm{mol} / \mathrm{L}$ for experimental group and $1.3 \pm 0.04 \mu \mathrm{mol} / \mathrm{L}$ for placebo). SOD increased significantly after supplementation $(\sim 14 \% ; P=0.0022)$, while no change occurred in the placebo group (Figure 2B). The improvement in oxidative stress markers and antioxidant capacity observed in the experimental group was accompanied by significant increases in both serum vitamin A (25\%) and vitamin E (65\%). Vitamin A did not change in the placebo group, and vitamin $\mathrm{E}$ showed a significant increase in this group $(\sim 14 \%)$. However, this increase was much lower than that observed in the experimental group.
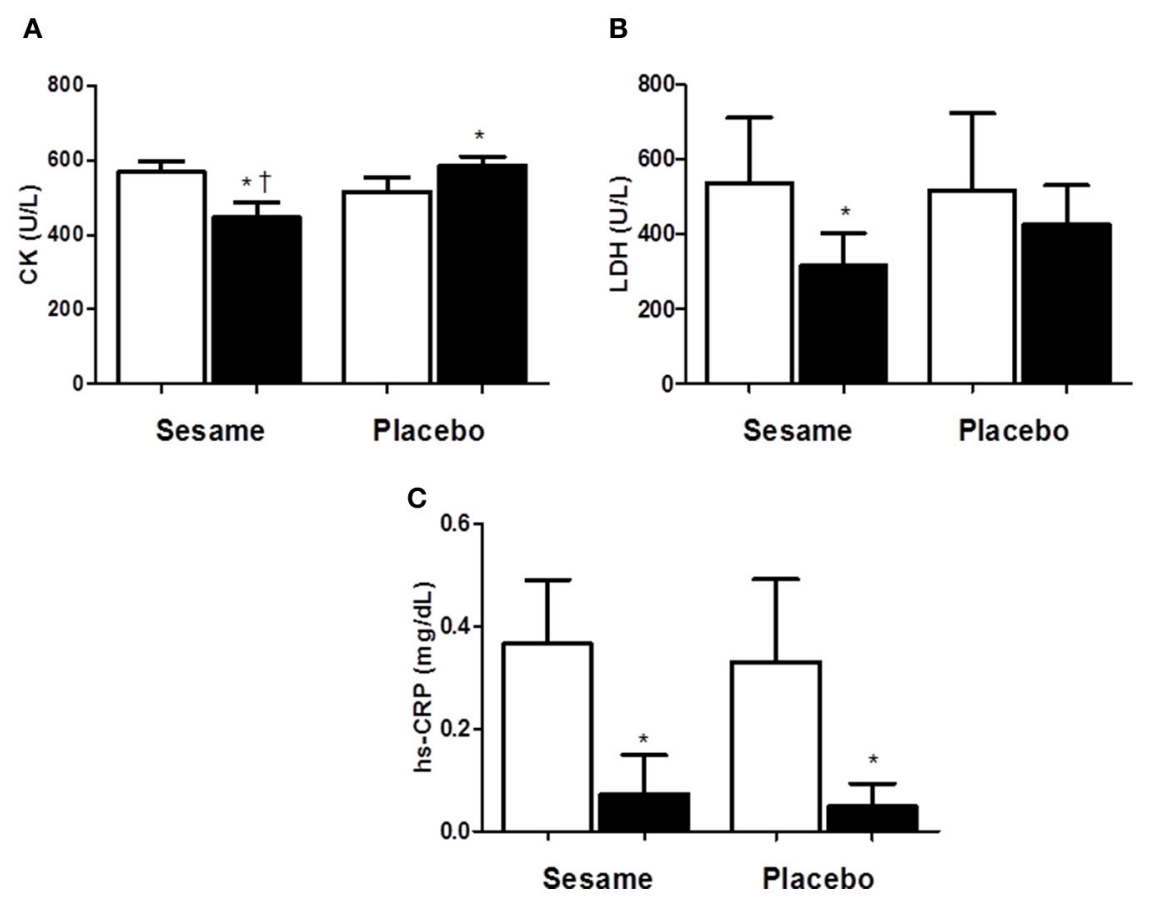

FIGURE 1 | Effect of sesame paste supplementation (Sesamum indicum L.) on muscle damage markers (CK and LDH-A, B) and systemic inflammation (hs-CRP, C). Data are expressed as average \pm standard deviation. ${ }^{*}$ Indicates intra-group differences between pre- and post-supplementation; ${ }^{\dagger}$ Indicates difference between groups at the end of the study (two-way ANOVA with Tukey's test). CK, creatinequinase enzyme; LDH, lactate dehydrogenase enzyme; hs-CRP, highly sensitive $\mathrm{C}$ reactivate protein. 
Thus, the final vitamin A and $\mathrm{E}$ values were significantly higher in the experimental group than in the placebo group $(P=0.0009$ and $P=0.0005$ to vitamin A and E, respectively; Figures 2C,D).

The experimental group showed significant increases in aerobic capacity $(\sim 17 \%)$ and $\mathrm{VO}_{2}$ peak speed $(\sim 10 \%)$, as shown in Figures 3A,B. No change was observed in the placebo group. However, the final values for these two variables did not differ between groups.

\section{DISCUSSION}

Biochemical markers commonly used for diagnosis of physiological state (Meeusen et al., 2013) and laboratorial reference parameters indicated physiological imbalance at the beginning of the study for the status of CK and $\mathrm{LDH}$ baseline levels. The athletes were already in the final months of a season, which explains the wear found present at the start of this study. This wear is commonly observed at the end of the season in soccer players (Handziski et al., 2006; Silva et al., 2014).

The observed deficiency in intake of vitamins $\mathrm{A}$ and $\mathrm{E}$ is probably related to the deficient intake of foods considered good sources for these vitamins by Brazilian adolescents (Tureck et al., 2013). After 28-day supplementation with the sesame paste, these two antioxidant vitamins ( $A$ and $E$ ) increased in the serum of the athletes, supporting the reported nutritional benefits of sesame (Anilakumar et al., 2010). The increase of
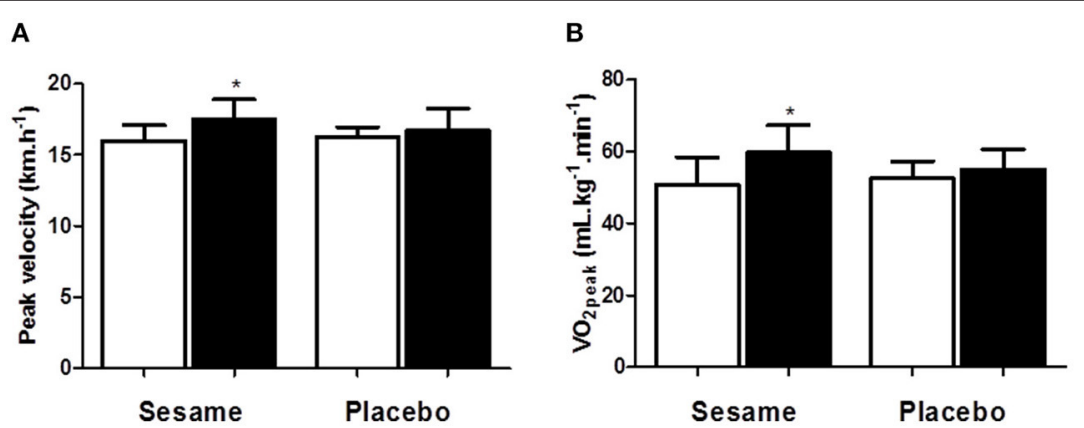

FIGURE 2 | Maximal aerobic capacity (A) and peak aerobic capacity speed (B) before and after supplementation with sesame paste (Sesamum indicum L.) or placebo. Data are expressed as average \pm standard deviation. *Indicates intra-group differences between pre- and post-supplementation (two-way ANOVA with Tukey's test).

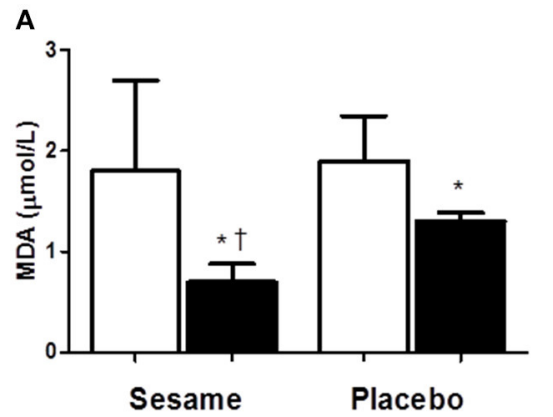

C

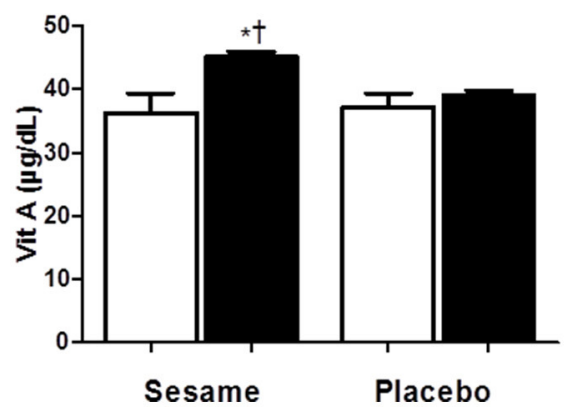

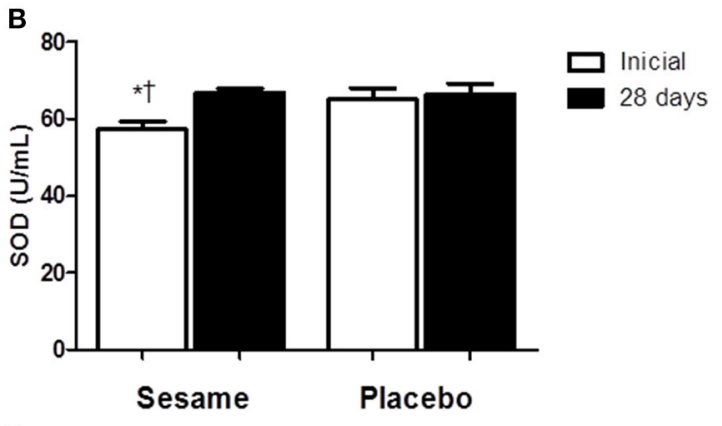

D

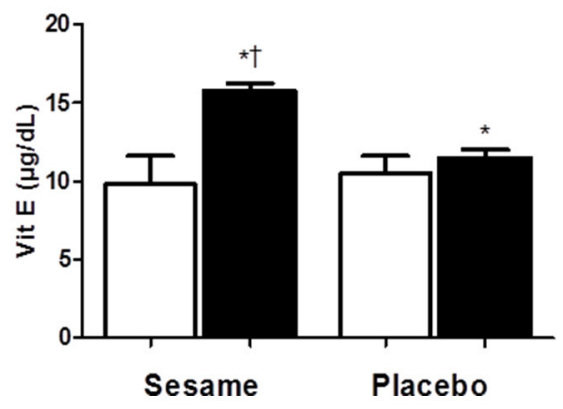

FIGURE 3 | Effect of sesame paste supplementation (Sesamum indicum L.) on lipid peroxidation (A) and enzymatic (SOD, B) and non-enzymatic antioxidants (vitamin A and E-C,D). Data are expressed as average \pm standard deviation. ${ }^{*}$ Indicates intra-group differences between pre- and post-supplementation; ${ }^{\dagger}$ Indicates difference between groups at the end of the study (Two-way ANOVA with Tukey's test). MDA, malondialdehyde; SOD, superoxide dismutase enzyme; Vit, vitamin. 
serum vitamin E after sesame seed supplementation has been reported in rats (Yamashita et al., 1995; Hanzawa et al., 2013). It has been suggested that sesame lignans regulate vitamin $\mathrm{E}$ metabolism by inhibiting tocopherol catabolism (Uchida et al., 2007; Hanzawa et al., 2013). However, other factors related to the effects of training on athlete's metabolism could have influenced the increase of vitamin $\mathrm{E}$, once it was also observed in placebo group.

The antioxidant activity of sesame seeds has been attributed to a synergistic effect of vitamin $\mathrm{E}$ with lignans that act to detoxify the hydroxyl radicals, inducing a reduction in lipid peroxidation and, consequently, in MDA level (Namiki, 2007; Askari et al., 2012). At the present time, we do not have explanations to justify the reduction of MDA serum levels in placebo group at the end of the study period. However, considering that this reduction was accompanied by an increase in vitamin $\mathrm{E}$, it could be related to a decrease in lipid peroxidation. The inversely proportional relation among vitamins $\mathrm{A}$ and $\mathrm{E}$ and lipid peroxidation is wellknown. The modulation of oxidizing agents production by intake of antioxidant vitamins (e.g., vitamins A and E) was previously described (Peuchant et al., 1997).

The reduction of the inflammation marker hs-CRP could be related to the antioxidant activity because the oxidative stress directly associated with the inflammatory response (Haddad, 2002). Finally, ROS formation plays an important role in the etiology of muscle injury and the lipid peroxidation due to increased production of ROS can lead to the release of muscle constituents (Taghiyar et al., 2013). This set of interactions between inflammatory processes, oxidative stress, and muscle damage could partially explain the changes in markers of oxidative stress and muscle damage markers observed in athletes supplemented with sesame paste. Further studies with larger number of participants and additional markers could help to clarify these interactions.

Interestingly, the increase in maximal aerobic capacity occurred even without any aerobic-focused training; the athletes were in the final stage of the season, when training focuses on strength, speed and technical-tactical elements. In fact, reduced aerobic capacity or absence of gains (but no increase) is expected in the final half of a soccer season (Kalapotharakos et al., 2011). Previous study reported an

\section{REFERENCES}

Alipoor, B., Haghighian, M. K., Sadat, B. E., and Asghari, M. (2012). Effect of sesame seed on lipid profile and redox status in hyperlipidemic patients. Int. J. Food Sci. Nutr. 63, 674-678. doi: 10.3109/09637486.2011.652077

Anilakumar, K. R., Pal, A., Khanum, F., and Bawa, A. S. (2010). Nutritional, medicinal and industrial uses of sesame (Sesamum indicum L.) seeds - an overview. Agric. Conspec. Sci. 75, 159-168. Available online at: http://hrcak.srce. $\mathrm{hr} / 66001$

Aptekmann, N. P., and Cesar, T. B. (2010). Orange juice improved lipid profile and blood lactate of overweight middle-aged women subjected to aerobic training. Maturitas 67, 343-347. doi: 10.1016/j.maturitas.2010.07.009

Askari, G., Ghiasvand, R., Feizi, A., Ghanadian, S. M., and Karimian, J. (2012). The effect of quercetin supplementation on selected markers of inflammation and oxidative stress. J. Res. Med. Sci. 17, 637-641. improved aerobic capacity concomitant with reduction of oxidative stress markers in runners supplemented with grape juice (Toscano et al., 2015). However, here it is not yet clear by which mechanisms reducing oxidative stress or systemic inflammation contributes to better aerobic capacity.

\section{CONCLUSION}

The results suggest that sesame consumption could be a nutritional strategy to improve the aerobic capacity of soccer players and to reduce important markers of oxidative stress and muscle damage.

\section{ETHICS STATEMENT}

This study was carried out in accordance with the recommendations of Resolution 196/96 of the Brazilian National Health Council (CNS) with written informed consent from all subjects. All subjects gave written informed consent in accordance with the Declaration of Helsinki. The protocol was approved by the Committee on Ethical Research Involving Humans Beings of the Federal University of Paraíba (João Pessoa, Brazil) under the Process Number 04308612.2.000 0.5183 .

\section{AUTHOR CONTRIBUTIONS}

CB participated in elaboration, execution and writing of the study. CO fulfilled the analyzes of MDA and $\mathrm{CK}$, and has participated in the implementation of dietary survey. NM helped to prepare the products and monitoring of training. YS, WC, and $\mathrm{RC}$ performed the physical-chemical and microbiological characteristics of the product. ACS offered all technical and scientific support about cultivating sesame that was used in the study. MM, ASS, PD, and VB participated in the elaboration, coordination or writing of the study.

\section{ACKNOWLEDGMENTS}

Thank CAPES-Brazil for a scholarship to the first author CB.
Association of Official Analytical Chemists (AOAC) (2012). Official Methods of Analysis. 19th Edn. Washington, DC: Association of Official Analytical Chemists.

Bruce, R. A., Kusumi, F., and Hosmer, D. (1973). Maximal oxygen intake and nomographic assessment of functional aerobic impairment in cardiovascular disease. Am. Heart J. 85, 546-562. doi: 10.1016/0002-8703(73)90502-4

Goldstein, D. S., and Kopin, I. J. (2007). Evolution of concepts of stress. Stress 10, 109-120. doi: 10.1080/10253890701288935

Haddad, J. J. (2002). Antioxidant and prooxidant mechanism in the regulation of $\operatorname{redox}(\mathrm{y})$-sensitive transcription factors. Cell. Signal. 14, 879-897. doi: 10.1016/S0898-6568(02)00053-0

Haghighian, M. K., Alipoor, B., Mahdavi, A. M., Sadat, B. E., Jafarabadi, M. A., and Moghaddam, A. (2015). Effects of sesame seed supplementation on inflammatory factors and oxidative stress biomarkers in patients with knee osteoarthritis. Acta Med. Iran 53, 207-213. 
Handziski, Z., Maleska, V., Petrovska, S., Nikolik, S., Mickoska, E., Dalip, M., et al. (2006). The changes of ACTH, cortisol, testosterone and testosterone/cortisol ratio in professional soccer players during a competition half-season. Bratisl. Med. J. 107, 259-263.

Hanzawa, F., Nomura, S., Sakuma, E., Uchida, T., and Ikeda, S. (2013). Dietary sesame seed and its lignan, sesamin, increase tocopherol and phylloquinone concentrations in male rats. J. Nutr. 143, 1067-1073. doi: $10.3945 /$ jn.113.176636

Institute of Medicine (2004). Dietary Reference Intakes for Vitamin A and E. Washington, DC: The National Academies Press. Available online at: https:// fnic.nal.usda.gov/dietary-guidance/dietary-reference-intakes/dri-nutrientreports (Accessed June 15, 2016).

Kalapotharakos, V. I., Ziogas, G., and Tokmakidis, S. P. (2011). Seasonal aerobic performance variations in elite soccer players. J. Strength Cond. Res. 25, 1502-1507. doi: 10.1519/JSC.0b013e3181da85a9

Kamal-Eldin, A., Moazzami, A., and Waxhi, S. (2011). Sesame seed lignans: potent physiological modulators and possible ingredients in functional foods \& nutraceuticals. Recent Pat. Food Nutr. Agric. 3, 17-29. doi: 10.2174/2212798411103010017

Kanu, P. J., Bahsoon, J. Z., Kanu, J. B., and Kandeh, J. B. A. (2010). Nutraceutical importance of sesame seed and oil: a review of the contribution of their lignans. J. Biomed. Res. 2, 4-16. doi: 10.4314/sljbr.v2i1.56583

Meeusen, R., Duclos, M., Foster, C., Fry, A., Gleeson, M., Nieman, D., et al. (2013). Prevention, diagnosis, and treatment of the overtraining syndrome: joint consensus statement of the European College of Sport Science and the American College of Sports Medicine. Med. Sci. Sports Exerc. 45, 186-205. doi: 10.1249/MSS.0b013e318279a10a

Miranda-Vilela, A. L., Pereira, L. C., Gonçalves, C. A., and Grisolia, C. K. (2009). Pequi fruit (Caryocar brasiliense Camb.) pulp oil reduces exercise-induced inflammatory markers and blood pressure of male and female runners. Nutr. Res. 29, 850-858. doi: 10.1016/j.nutres.2009.10.022

Namiki, M. (2007). Nutraceutical functions of sesame: a review. Crit. Rev. Food Sci. Nutr. 47, 651-673. doi: 10.1080/10408390600919114

Peuchant, E., Delmas-Beauvieux, M. C., Dubourg, L., Thomas, M., Perromat, A., Aparicio, M., et al. (1997). Antioxidant effects of a supplemented very low protein diet in chronic renal failure. Free Radic. Biol. Med. 22, 313-320. doi: 10.1016/S0891-5849(96)00282-1

Silva, J. R., Rebelo, A., Marques, F., Pereira, L., Seabra, A., and Ascensão, A. (2014). Biochemical impact of soccer: an analysis of hormonal, muscle damage, and redox markers during the season. J. Appl. Physiol. Nutr. Metab. 39, 432-438. doi: 10.1139/apnm-2013-0180
Smith, L. L. (2004). Tissue trauma: the underlying cause of overtraining syndrome? J. Strength Cond. Res. 18, 185-193. doi: 10.1519/00124278-200402000-00028

Suwimol, S., Wiroj, J., Walin, W. K., Natchapon, J., Pathamaporn, H. U., and Auranun, S. A. (2012). Effects of sesame seeds consumption on serum cholesterol and oxidative status in hypercholesterolemia. Food Pub. Health 2 , 193-196. doi: 10.5923/j.fph.20120206.02

Taghiyar, M., Darvishi, L., Askari, G., Feizi, A., Hariri, M., Mashhadi, N. S., et al. (2013). The effect of vitamin $C$ and $E$ supplementation on muscle damage and oxidative stress in female athletes: a clinical trial. Int. J. Prev. Med. 4 16-23.

Templar, J., Kon, S. P., Milligan, T. P., Newman, D. J., and Raftery, M. J. (1999). Increased plasma malondialdehyde levels in glomerular disease as determined by a fully validated HPLC method. Nephrol. Dial. Transplant14, 946-951. doi: $10.1093 / \mathrm{ndt} / 14.4 .946$

Toscano, L. T., Tavares, R. L., Toscano, L. T., Silva, C. S. O., Almeida, A. E. M., Biasoto, A. C. T., et al. (2015). Potential ergogenic activity of grape juice in runners. Appl. Physiol. Nutr. Metab. 40, 899-906. doi: 10.1139/apnm-2015-0152

Tureck, C., Gesser, C. V. G., Peralta, R. M., and Koehlein, E. A. (2013). Intakes of antioxidant vitamins and minerals in the Brazilian diet. Nutr. Clin. Diet Hosp. 33, 30-38. doi: 10.12873/333Braziliandiet

Uchida, T., Ichikawa, T., Abe, C., Yamashita, K., and Ikeda, S. (2007). Dietary sesame seed decreases urinary excretion of alpha- and gamma-tocopherol metabolites in rats. J. Nutr. Sci. Vitaminol. 53, 372-376. doi: 10.3177/jnsv. 53.372

Winsley, R., and Matos, N. (2011). Overtraining and elite young athletes. Med. Sport Sci. 56, 97-105. doi: 10.1159/000320636

Yamashita, K., Iizuka, Y., Imai, T., and Namiki, M. (1995). Sesame seed and its lignans produce marked enhancement of vitamin $\mathrm{E}$ activity in rats fed a low alpha-tocopherol diet. Lipids 30, 1019-1028. doi: 10.1007/BF02536287

Conflict of Interest Statement: The authors declare that the research was conducted in the absence of any commercial or financial relationships that could be construed as a potential conflict of interest.

Copyright (C) 2017 Barbosa, Silva, de Oliveira, Massa, de Sousa, da Costa, Silva, Delatorre, Carvalho, Braga and Magnani. This is an open-access article distributed under the terms of the Creative Commons Attribution License (CC BY). The use, distribution or reproduction in other forums is permitted, provided the original author(s) or licensor are credited and that the original publication in this journal is cited, in accordance with accepted academic practice. No use, distribution or reproduction is permitted which does not comply with these terms. 